03

\title{
Волоконная система генерации импульсов высокой спектральной плотности
}

\author{
(C) А.С. Абрамов ${ }^{1}$, И.О. Золотовский ${ }^{1}$, Д.А. Коробко ${ }^{1, \uparrow, ~ А . А . ~ Ф о т и а д и ~}{ }^{1,2}$ \\ ${ }^{1}$ Ульяновский государственный университет, \\ 432017 Ульяновск, Россия \\ ${ }^{2}$ Université de Mons, \\ B7000 Mons, Belgium \\ ๑ e-mail: korobkotam@rambler.ru
}

Поступила в редакцию 17.10.2017 г.

\begin{abstract}
Предложена каскадная оптоволоконная система генерации импульсов высокой спектральной плотности, основанная на эффекте нелинейной спектральной компрессии. Показано, что форма огибающей импульса существенно влияет на степень компрессии его спектра, при этом максимум компрессии достигается для параболических импульсов. Элементами каскадной системы являются волокно с убывающей по длине нормальной дисперсией, обеспечивающее приближение огибающей импульса к параболической форме, дифракционные решетки и волоконный спектральный компрессор. При помощи численного моделирования в работе определены значения параметров элементов каскада, при выборе которых достигается максимальная спектральная плотность излучения, источником которого является субпикосекундный лазерный импульс средней энергии.
\end{abstract}

DOI: $10.21883 / \mathrm{OS} .2018 .03 .45656 .234-17$

\section{Введение}

Разработка систем генерации лазерного излучения с высокой спектральной плотностью является весьма актуальной задачей, так как имеет ряд важных приложений - например, генерацию кратных гармоник и конверсию частот в нелинейных средах $[1,2]$, эффективное усиление сигнала в активной резонансной среде и резонансное облучение среды (например, с целью выделения изотопов $[3,4])$. Высокое прикладное значение актуализирует развитие направления, связанного с разработкой волоконных излучателей высокой спектральной плотности. Компактность, надежность, высокое качество пучка делают системы на основе оптических волокон крайне привлекательными для использования, однако, волоконные лазерные источники высокой спектральной чистоты обычно имеют достаточно невысокую мощность [5], а при усилении нелинейные процессы в волокне приводят к значительному уширению спектра [6]. В настоящей работе предлагается альтернативный подход, основанный на нелинейной спектральной компрессии (СК) мощного импульсного излучения волоконного лазера, обеспечивающий получение импульсов с высокой спектральной яркостью.

Нелинейная СК - известный эффект, проявляющийся за счет фазовой самомодуляции (ФСМ) лазерного импульса с отрицательным чирпом, распространяющегося в нелинейном световоде $[7,8]$. Этот эффект заключается в том, что на определенной длине распространения в волокне ФСМ практически полностью компенсирует отрицательный чирп, при этом спектр импульса обладает минимальной шириной. При разработке волоконного генератора высокой спектральной плотности в качестве источника мы предлагаем воспользоваться стандартным волоконным субпикосекундным импульсным лазером со средней энергией импульса, излучение которого затем будет спектрально сжато. Современное развитие волоконных лазеров позволяет использовать для этой цели источники различных диапазонов (1050, 1550, $1950 \mathrm{~nm}$ и др.) с длительностью импульса порядка $0.2-1$ ps и энергией не менее $1 \mathrm{~nJ}[9,10]$. В настоящей работе предложен вариант схемы СК подобных импульсов, подходящий для любого спектрального диапазона, соответствующего излучению волоконных лазеров, и позволяющий достичь высоких уровней спектральной яркости, необходимых в приложениях.

\section{1. Модель волоконного спектрального компрессора}

Рассматривать распространение импульсов в нелинейном волокне будем на основе нелинейного уравнения Шредингера (НУШ) для комплексной амплитуды [11]:

$$
\frac{\partial A}{\partial z}-\frac{i \beta_{2}}{2} \frac{\partial^{2} A}{\partial t^{2}}+i \gamma|A|^{2} A=0 .
$$

Здесь $z$ - продольная координата, $t-$ время в сопутствующей системе координат, $\beta_{2}-$ коэффициент дисперсии групповых скоростей (ДГС) волокна, $\gamma$ параметр керровской нелинейности. Для простоты и ясности изложения пренебрежем в этом разделе потерями в волокне и зависимостью $\beta_{2}$ от длины волны, т. е. вкладами дисперсий третьего и более высоких порядков. Это возможно сделать в том случае, если длина волокна существенно меньше $L_{3 \mathrm{D}}=2 \tau^{3} / \beta_{3}$, где $\tau$ - длительность 


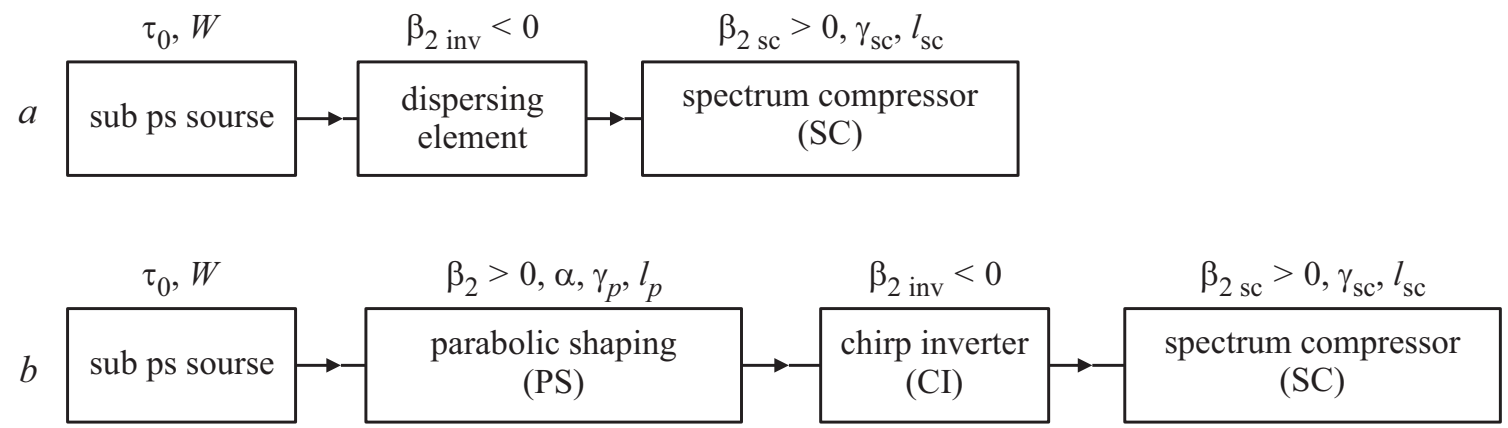

Рис. 1. Схемы для спектральной компрессии лазерных импульсов ( $a-$ без $P S$-элемента, $b-\mathrm{c} P S$-элементом).

импульса, подаваемого на вход спектрального компрессора, $\beta_{3}$ - дисперсия третьего порядка. Это условие выполняется для импульсов длительностью 10 ps и более, распространяющихся в волоконных световодах длиной менее $1000 \mathrm{~m} \mathrm{c}$ величиной $\beta_{3}<10^{-1} \mathrm{ps}^{3} / \mathrm{m}$. В нашем случае реализуется именно эта ситуация.

Варианты систем для компрессии спектров лазерных импульсов показаны на рис. 1. Здесь же введены соответствующие обозначения для параметров дисперсии групповых скоростей $\beta_{2}$ и нелинейности $\gamma$, характеризующие каждый элемент системы. В стандартной схеме СК (рис. 1,a) на первом этапе изначально частотно немодулированный импульс проходит линейный диспергирующий элемент (dispersing element), где он приобретает отрицательный линейный чирп. Наилучшим образом этот процесс можно осуществить при помощи пары дифракционных решеток. Амплитуда огибающей импульса после прохождения этого элемента может быть записана в виде

$$
A(0, t)=a(t) \exp \left(i C t^{2}\right)
$$

где $C<0-$ скорость частотной модуляции. На следующем и последнем этапе импульс с отрицательной частотной модуляцией инжектируется в нелинейное волокно. Там при распространении импульс подвергается воздействию ФСМ, которая придает импульсу частотную модуляцию, противоположную исходной, т.е. в фазе импульса появляется дополнительное слагаемое, пропорциональное длине распространения $z-\gamma|a|^{2} z$. В результате частотная модуляция импульса приобретает достаточно сложный вид, зависящий от формы огибающей импульса, начальной частотной модуляции и длины распространения

$$
\varphi(t)=C t^{2}-\gamma|a|^{2} z
$$

Ширина спектра импульса, обладающего высокой частотной модуляцией, $\sim \varphi^{\prime}(t)$. Таким образом, на определенной длине волокна за счет компенсации отрицательного чирпа можно добиться минимальной частотной ширины спектра, т.е. его максимальной компрессии. Очевидно, что качество компрессии зависит от формы огибающей $a(t)$. Можно показать, что параболический вид огибающей импульса

$$
a(t)=\sqrt{P_{0}\left(1-\frac{t^{2}}{\tau_{\mathrm{p}}^{2}}\right)}, \quad|t| \leq \tau_{\mathrm{p}}, \quad a=0, \quad|t|>\tau_{\mathrm{p}}
$$

обеспечивает наилучший результат $[12,13]$, где $P_{0}-$ пиковая мощность. Действительно, в бездисперсионном случае $\beta_{2}=0$ на некоторой дистанции $z=L$, частотная модуляция может быть полностью скомпенсирована

$$
\varphi(t)=C_{0} t^{2}-\gamma P_{0}\left(1-\frac{t^{2}}{\tau_{\mathrm{p}}^{2}}\right) L=-\gamma P_{0} L=\mathrm{const},
$$

т.е. импульс становится спектрально ограниченным $\varphi^{\prime}(t) \equiv 0$, при этом достигается минимальная ширина спектра. В приближении стандартного вариационного метода, согласно которому распространяющийся импульс сохраняет форму огибающей, несложно провести оценку влияния ДГС на длину СК [14]. Результаты показывают, что по отношению к бездисперсионному случаю СК значительно быстрее проходит в волокнах с нормальной ДГС. Вследствие сопутствующей временной компрессии импульса минимальная ширина спектра $\Delta \Omega_{\min }$, достижимая при компрессии в волокне с нормальной ДГС, несколько больше величины $\Delta \Omega_{\min }$, получаемой в бездисперсионном случае (при $\beta_{2}=0$ ).

Рассматривая лазерные источники с другим профилем импульса, например гауссовым, отметим, что в отличие от случая параболической огибающей их спектральная компрессия сопровождается появлением искажений ФСМ лишь частично либо локально компенсирует начальный чирп, что характеризуется появлением „крыльев“ и/или „пьедестала“ в сжатом спектре импульса и значительно снижает эффективность процесса. На основании вышеизложенного предлагается модернизировать схему стандартной СК, добавив в ее состав дополнительный $P S$-элемент (parabolic shaping), обеспечивающий плавное преобразование начальной формы огибающей импульса к параболе (рис. $1, b)$. Сделать это возможно, например, при использовании современных технологий оптического процессинга - формированием импульса с заданным профилем огибающей и необходимой частотной модуляцией. В качестве таких элементов могут 
Параметры импульса и входящих в схему элементов

\begin{tabular}{|c|c|c|c|c|c|c|c|c|c|c|c|}
\hline № & \multicolumn{3}{|c|}{1} & \multicolumn{4}{|c|}{2} & 3 & \multicolumn{3}{|c|}{4} \\
\hline Элемент & \multicolumn{3}{|c|}{ Источник излучения } & \multicolumn{4}{|c|}{$\begin{array}{c}\text { Волокно с плавно } \\
\text { убывающей дисперсией } \\
\text { и постоянной нелинейностью }\end{array}$} & $\begin{array}{c}\text { Дифракционные } \\
\text { решетки }\end{array}$ & \multicolumn{3}{|c|}{$\begin{array}{c}\text { Волокно } \\
\text { с постоянной дисперсией } \\
\text { и нелинейностью }\end{array}$} \\
\hline Стадия & \multicolumn{3}{|c|}{ Ввод излучения } & \multicolumn{4}{|c|}{ Параболизация огибающей } & Инверсия чирпа & \multicolumn{3}{|c|}{ Компрессия спектра } \\
\hline Параметры & $\tau_{0}, \mathrm{ps}$ & $\begin{array}{c}\text { Пиковая } \\
\text { мощность, W }\end{array}$ & $\begin{array}{c}\text { Начальный } \\
\text { чирп, } C_{0}, \mathrm{~s}^{-2}\end{array}$ & $\begin{array}{c}\beta_{20} \\
\mathrm{ps}^{2} / \mathrm{km}\end{array}$ & $\begin{array}{c}\alpha, \\
\mathrm{m}^{-1}\end{array}$ & $\begin{array}{c}\gamma_{\mathrm{p}}, \\
(\mathrm{W} \cdot \mathrm{km})^{-1}\end{array}$ & $\begin{array}{l}l_{\mathrm{p}} \\
\mathrm{m}\end{array}$ & $\beta_{2 \mathrm{inv}}, \mathrm{ps}^{2}$ & $\begin{array}{c}\beta_{2 \mathrm{sc}} \\
\mathrm{ps}^{2} / \mathrm{km}\end{array}$ & $\begin{array}{c}\gamma_{\mathrm{sc}} \\
(\mathrm{W} \cdot \mathrm{km})^{-1}\end{array}$ & $\begin{array}{l}l_{\mathrm{sc}}, \\
\mathrm{m}\end{array}$ \\
\hline Значения & 0.2 & 3600 & 0 & 84 & 0.5 & 3 & 100 & -0.22 & 5 & 7 & 225 \\
\hline
\end{tabular}

быть рассмотрены программируемые фазовые маски на жидких кристаллах или программируемые акустооптические фильтры [15]. Однако использование таких сложных и дорогостоящих элементов не является обязательным. Как известно, параболический профиль огибающей импульс приобретает автоматически при прохождении в нелинейном волокне с гиперболически убывающей нормальной дисперсией [16]. Этот эффект связан с тем, что распространение импульса в световоде с убывающей нормальной ДГС вследствие относительного роста влияния нелинейности эквивалентно распространению в усиливающей среде с бесконечно широкой полосой усиления. Его результатом становится образование параболического симиляритонного импульса (т.е. изменяющегося „самоподобно“) с линейным чирпом [17]. Технологически световоды с изменяющейся ДГС и постоянным при этом параметром нелинейности волокна $\gamma$ могут быть изготовлены, например, на основе волокон c W-профилем показателя преломления в поперечном сечении. При их вытяжке можно достичь существенного изменения ДГС за счет небольшой вариации диаметра волокна, при этом площадь моды и коэффициент нелинейности вдоль такого с большой степенью точности можно считать постоянными [18]. Дополняя систему таким элементом, возможно переформировать огибающую и добиться улучшения качества СК на финальном этапе.

\section{2. Численный анализ}

В этом разделе будут представлены результаты численного моделирования предлагаемых систем СК. В качестве исходного импульса используется спектрально ограниченный гауссов импульс субпикосекундной длительности. Характеристики входящего в каскадную схему импульса, а также дисперсионные и нелинейные параметры элементов системы приведены в таблице.

На рис. 2, а показана схема компрессии спектра исходного гауссова импульса в соответствии со стандартной схемой (рис. 1, $a$ ). На первом этапе импульсу придается отрицательный чирп, затем он инжектируется в $S C$-элемент, где и происходит нелинейная компрессия спектра. Отмечаем, что из-за отличия в исходной форме

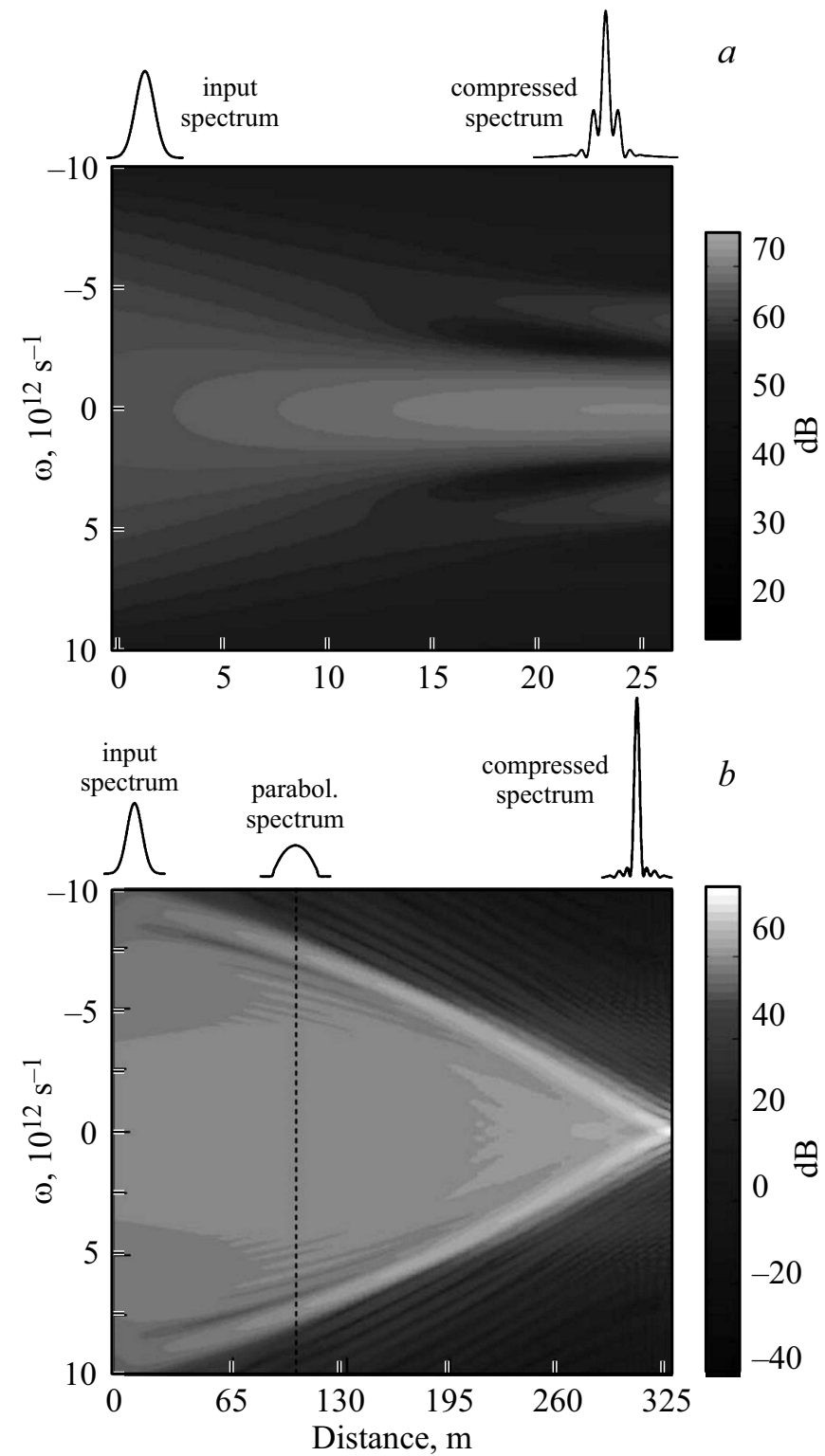

Рис. 2. Эволюции спектров гауссова импульса в волоконных каскадных схемах компрессии без $P S$-элемента $(a)$ и в схемax, содержащих данный элемент $(b)$. Схематично указано видоизменение огибающей спектра импульса по мере его прохождения стадий каскада. 
огибающей для сжатого спектра характерно присутствие „крыльев“, сопровождающих главный пик, что в результате приводит к низкой итоговой спектральной плотности (см. ниже рис. 5).

Для сравнения на рис. 2, 6 приведена эволюция спектра огибающей импульса по мере его прохождения по модернизированному волоконному каскаду. Штриховой линией обозначена граница между элементом, отвечающим за параболизацию огибающей (PS-элемент), и компрессором спектра ( $S C$-элемент), представленными в виде волоконных световодов с различными значениями дисперсии и нелинейности. По мере прохождения импульса через $P S$-элемент наблюдается плавное уширение его спектра, и на длине волокна $l_{\mathrm{p}}=100 \mathrm{~m}$ форма огибающей импульса приближается к параболической. Спектральная плотность при этом снижается практически в 4 раза, а пиковая мощность импульса уменьшается более чем в 50 раз (рис. 3). Подготовленный импульс проходит через дифракционные решетки, где его чирп инвертируется, и далее в $S C$-волоконном элементе с постоянной нормальной дисперсией осуществляется спектральная компрессия импульса за счет постепенной компенсации отрицательной частотной модуляции. При выбранных расчетных параметрах максимальная компрессия спектра реализуется в волокне длиной $l_{\mathrm{sc}}=225 \mathrm{~m}$. Численные оценки и более подробное описание каждого из элементов приведены ниже.

\subsection{PS-элемент}

На вход первого элемента рассматриваемой волоконной системы от источника излучения подается субпикосекундный импульс с гауссовой огибающей

$A(\tau)=A_{0} \exp \left[-\frac{1}{2}\left(\frac{t}{\tau_{0}}\right)^{2}\right], \quad \tilde{A}(\omega)=\frac{1}{2 \pi} \int_{-\infty}^{\infty} A(t) \mathrm{e}^{-i \omega t} d t$

Начальная длительность импульса $\tau_{0}=0.2 \mathrm{ps}$, пиковая мощность на входе $\left|A_{0}\right|^{2}=3600 \mathrm{~W}$, при этом импульс изначально не обладает частотной модуляцией.

$P S$-элемент представлен участком волокна с постоянным коэффициентом нелинейности $\gamma_{\mathrm{p}}$. Нормальная дисперсия на этом участке убывает по гиперболическому закону, который записывается в виде $\beta_{2}(z)=\beta_{20} /(1+\alpha z)$. Здесь $\beta_{20}-$ начальное значение дисперсии на входе в элемент, $\alpha$ - декремент снижения дисперсии. Численное моделирование эволюции огибающей импульса проводилось в рамках стандартного пошагового метода преобразований Фурье, заключающегося в последовательном учете дисперсии и нелинейности на каждом отдельно взятом малом участке волокна [11]. Как уже было отмечено, на выходе из $P S$-элемента наблюдается дисперсионное расплывание импульса, при этом его спектр нелинейно уширяется. Огибающая импульса приближается к параболическому виду, а частотная модуляция (чирп) соответственно приобретает линейный вид (рис. 3,4 , кривые 2 ).

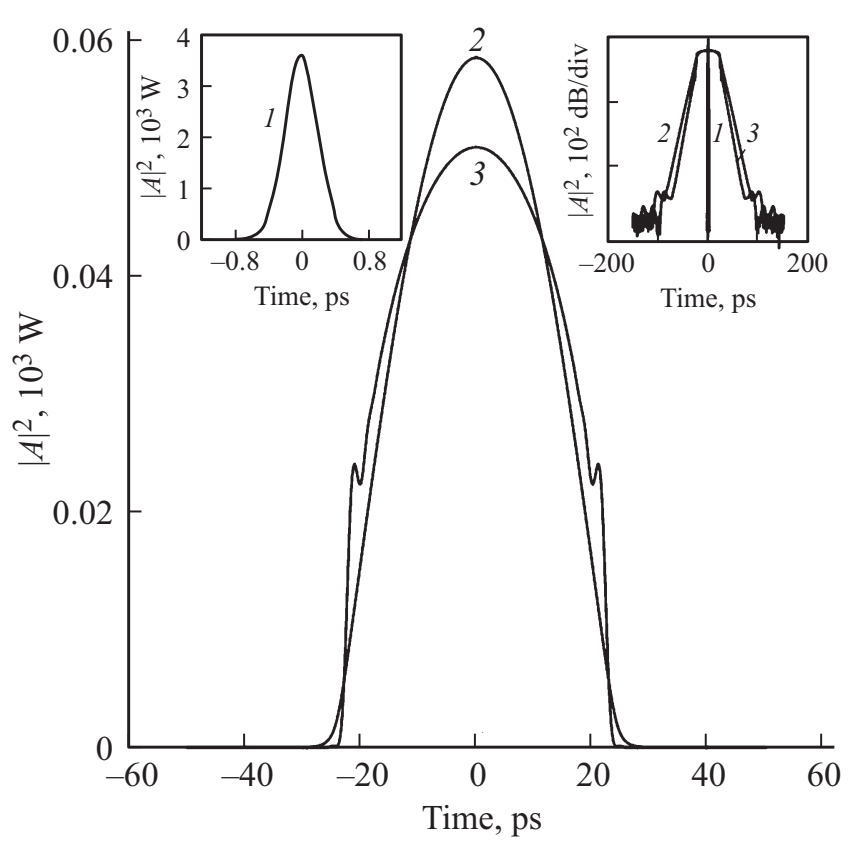

Рис. 3. Временнб́е профили входящего импульса (кривая 1), импульса, прошедшего стадию параболизации (кривая 2), импульса с инвертированным чирпом (кривая 3 ).

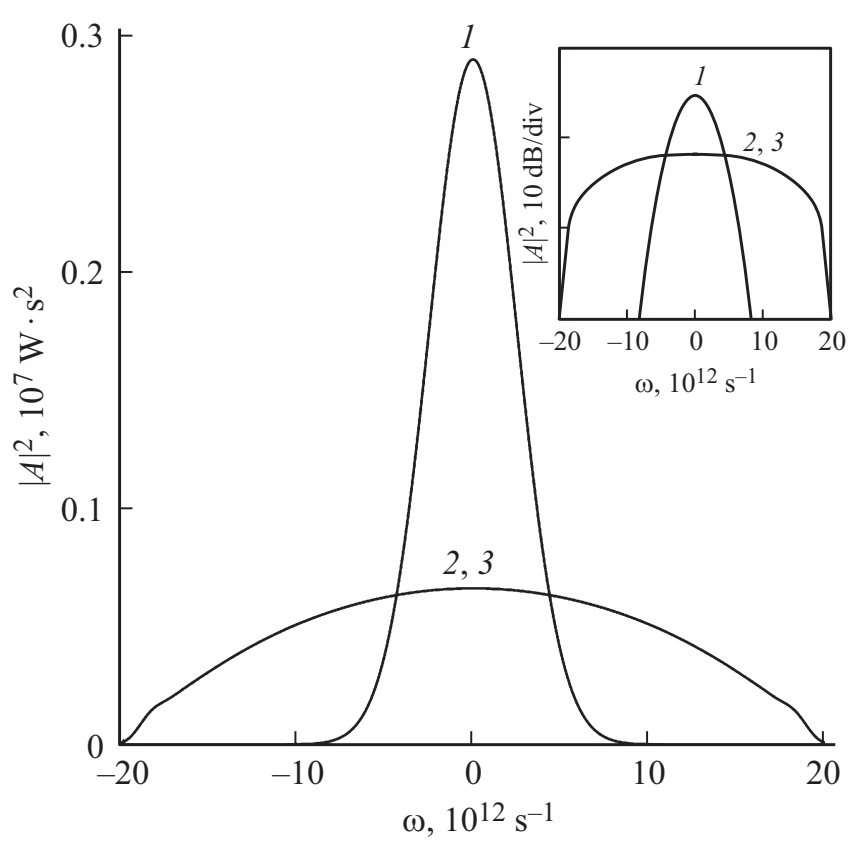

Рис. 4. Спектры входящего импульса (кривая 1) и спектры после стадии параболизации и инверсии чирпа (кривые 2,3). На вставке те же спектры в логарифмическом масштабе.

\section{2. Инвертор чирпа - CI-элемент}

После стадии параболизации импульса следует стадия, на которой импульсу придается отрицательная частотная модуляция (инверсия чирпа). В рассматриваемой схеме это реализуется с помощью пары дифракционных решеток, имеющих аномальную дисперсию. При этом 
величина дисперсии $\beta_{2 \text { inv }}$ подбирается таким образом, чтобы временной профиль огибающей импульса после стадии параболизации по возможности не искажался, а его ширина на полувысоте при инверсии оставалась постоянной (рис. 2, кривые 2,3).

$$
\tilde{A}_{\text {inv }}(z, \omega)=\tilde{A}(z, \omega) \exp \left(\frac{i}{2} \beta_{2 \text { inv }} \omega^{2}\right) .
$$

\section{3. $S C$-элемент}

Последним и основным элементом (SC-элемент) предлагаемой схемы является волокно, в котором отрицательная частотная модуляция компенсируется за счет воздействия фазовой самомодуляции. Длина участка волокна $-l_{\mathrm{sc}}$, при этом нормальная дисперсия групповых скоростей $\beta_{2 \mathrm{sc}}$ и нелинейность $\gamma_{\mathrm{sc}}$ постоянны по его длине. Численный расчет эволюции огибающих также проводился с помощью пошагового метода преобразований Фурье. На рис. 5 изображен спектр, прошедший все стадии ( $P S$ - и $S C$-элементы - кривая 3$)$. Здесь же для сравнения приведен начальный спектр гауссова импульса (кривая 1) и сжатый спектр, который получен из него по стандартной схеме (без стадии параболизации) (кривая 2). Отмечаем, что в этом случае компрессия спектра значительно менее эффективна. Более того, практически половина всей энергии импульса приходится на спектральные „крылья“, которые неизбежно будут утрачены при конечной узкополосной фильтрации сигнала. В спектре импульса, прошедшего предварительную параболизацию, подобные искажения

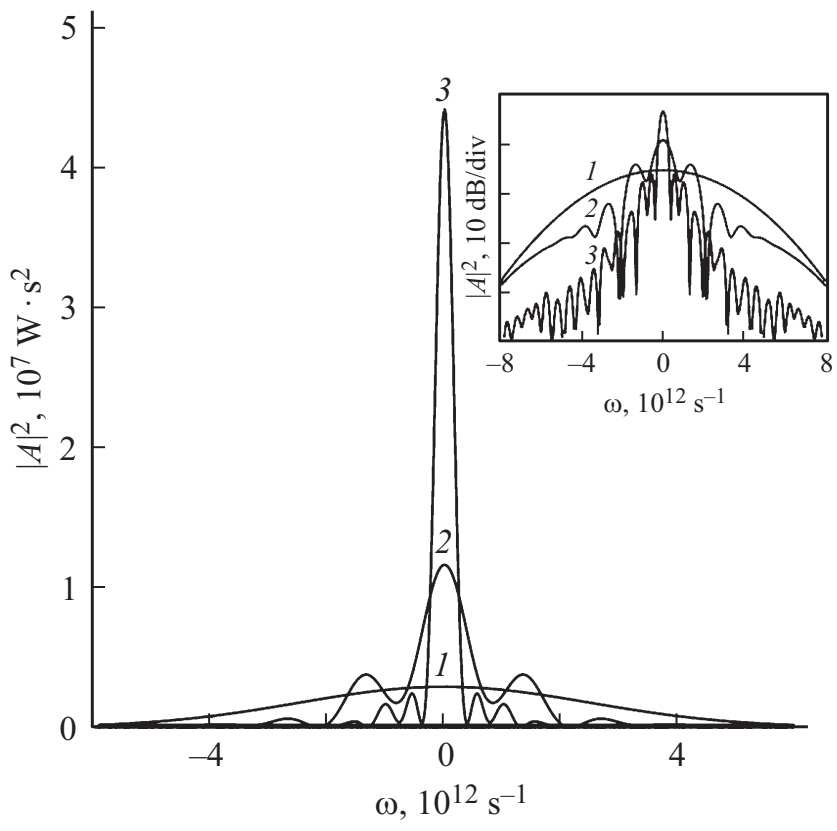

Рис. 5. Спектры входящего гауссова импульса (кривая 1), гауссова импульса, не прошедшего $P S$-элемент (кривая 2), и импульса, прошедшего стадию параболизации (3). На вставке - те же спектры в логарифмическом масштабе. выражены значительно меньше. Пиковое значение спектральной плотности на порядки превышает значения в сопутствующих локальных максимумах.

В результате отмечаем, что для сжатого спектра импульса, прошедшего $P S$-элемент, ширина спектра на полувысоте равна $\Delta \omega=0.336 \cdot 10^{12} \mathrm{~s}^{-1}$ при пиковой спектральной плотности $P_{\max }=4.41 \cdot 10^{7} \mathrm{~W} \cdot \mathrm{s}^{2}$ (кривая 3). В то же время эти величины для сжатого спектра импульса, не прошедшего волокно с убывающей по длине дисперсией, равны соответственно $\Delta \omega=1.33 \cdot 10^{12} \mathrm{~s}^{-1}$ и $P_{\max }=1.09 \cdot 10^{7} \mathrm{~W} \cdot \mathrm{s}^{2}$ (кривая 2). Таким образом, предварительное прохождение импульса через $P S$-элемент позволяет практически в 4 раза повысить эффективность нелинейной спектральной компрессии.

\section{Заключение}

В работе численно исследована нелинейная компрессия спектра субпикосекундного лазерного импульса средней энергии в оптоволоконной системе. Показано, что критически важным показателем качества итоговой компрессии является форма огибающей исходного импульса, при этом максимальная степень компрессии спектра достигается при параболической форме огибающей.

Предложена каскадная модель волоконной системы компрессии спектра с предварительным элементом, обеспечивающим переформирование огибающей и ее приближение к параболическому виду. Показано, что при значениях параметров, характерных для стандартных оптических одномодовых волокон, предложенная каскадная модель обеспечивает повышенное качество компрессии спектра (по сравнению со стандартной схемой СК). Предложенная модель может быть использована в ряде нелинейно-оптических приложений например, в конверсии частот и генерации кратных гармоник.

Работа выполнена при поддержке Министерства образования и науки Российской Федерации (Государственное задание № 3.3889.2017) и Российского научного фонда (проекты 16-42-02012 и 17-7210135).

\section{Список литературы}

[1] Wynands R., Coste O., Rembe C., Meschede D. // Opt. Lett. 1995. V. 20. P. $1095-1097$.

[2] Запорожченко Р.Г. // Опт. и спектр. 2003. Т. 95. № 6. C. $1043-1050$.

[3] Ткачев А.Н., Яковленко С.И. // Квант. электрон. 2003. Т. 33. C. 581-592.

[4] Макаров Г.Н. // УФН. 2015. Т. 185. С. 717-751.

[5] Ylä-Jarkko K.H., Grudinin A.B. // IEEE Photon. Technol. Lett. 2003. V. 15. P. 191.

[6] Singh S., Singh N. //Progress in Electromagnetics Research. 2007. V. 73. P. $249-275$.

[7] Stolen R.H., Lin C. // Phys. Rev. A. 1978. V. 17. P. 1448. 
[8] Planas S.A., Mansur N.P., Cruz C.B., Fragnito H.L. // Opt. Lett. 1993. V. 18. P. 699-701.

[9] Digonnet M.J. Rare-earth-doped fiber lasers and amplifiers, revised and expanded. CRC press, 2001.

[10] Tünnermann A., Schreiber T., Limpert J. // Appl. Opt. 2010. V. 49. P. $71-78$.

[11] Agrawal G. Nonlinear fiber optics. Springer, 2007. 530 p.

[12] Fatome J., Kibler B., Andresen E., Rigneault H., Finot C. // Appl. Opt. 2012. V. 51. P. 4547-4553.

[13] Boscolo S., Chaussard F., Andresen E., Rigneault H., Finot C. // Optics and Laser Technology. 2018. V. 99. P. 301-309. doi: 10.1016/j.optlastec.2017.08.033

[14] Zolotovskii I.O., Korobko D.A., Sysoliatin A.A., Fotiadi A.A. // J. Russian Laser Research. 2016. V. 37. P. 448-458.

[15] Monmayrant A., Weber S., Chatel B. // J. Physics B: Atomic, Molecular and Optical Physics. 2010. V. 43. P. 103001.

[16] Hirooka T., Nakazawa M. // Opt. Lett. 2004. V. 29. P. 498-500.

[17] Золотовский И.О., Коробко Д.А., Охотников О.Г., Семенцов Д.И., Сысолятин А.А., Фотиади А.А. // Опт. и спектр. 2013. T. 114. № 2. С. 286-286.

[18] Ахметиин У.Г., Богатырев В.А., Сенаторов А.К., Сысолятин А.А., Шалыгин М.Г. // Квант. электрон. 2003. Т. 33. C. $265-267$. 